\title{
Feasibility of a novel low-volume and sodium phosphate-free bowel preparation regimen for colon capsule endoscopy
}

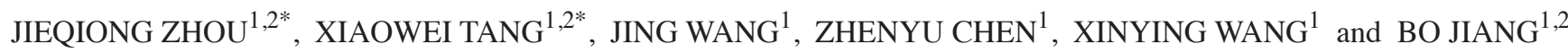 \\ ${ }^{1}$ Guangdong Provincial Key Laboratory of Gastroenterology, Department of Gastroenterology, Nanfang Hospital, \\ Southern Medical University, Guangzhou, Guangdong 510515; ${ }^{2}$ Department of Gastroenterology, \\ Beijing Tsinghua Chang Gung Hospital, Tsinghua University, Beijing 102218, P.R. China
}

Received May 11, 2016; Accepted April 7, 2017

DOI: $10.3892 /$ etm.2017.4664

\begin{abstract}
Bowel preparation regimens for colon capsule endoscopy are not yet standardized since they are not well optimized. The aim of the present study was to evaluate the feasibility of a novel low-volume and sodium phosphate-free bowel preparation regimen for colon capsule endoscopy. A total of 31 patients were prospectively enrolled. In the novel regimen, on the day prior to examination, a low-fiber diet was permitted, $5 \mathrm{mg}$ mosapride citrate was administered twice ( $1 \mathrm{~h}$ prior to lunch and supper) and 11 polyethylene glycol was administered in the evening. On the day of the examination, an additional 11 polyethylene glycol, $5 \mathrm{mg}$ mosapride citrate and $200 \mathrm{mg}$ simethicone were administered before capsule ingestion. Polyethylene glycol booster $(0.51)$ was administered twice, at 1 and $4 \mathrm{~h}$ following capsule ingestion. Colon cleansing levels, ileocecal valve transit time and completion rate were evaluated. A total of 29 patients were included in the final analysis, $90 \%$ of whom achieved adequate preparation of the overall colon. Ileocecal valve transit time was $2.35 \pm 0.82 \mathrm{~h}$ and completion rate was $79.3 \%$. The results revealed that the novel low-volume and sodium phosphate-free bowel preparation regimen for colon capsule endoscopy was feasible, with adequate colon cleansing and completion rates, and has the potential to be used as an alternative regimen.
\end{abstract}

\section{Introduction}

Colorectal cancer (CRC) is the second most common type of cancer in western countries and has exhibited an increasing

Correspondence to: Dr Bo Jiang or Dr Xinying Wang, Guangdong Provincial Key Laboratory of Gastroenterology, Department of Gastroenterology, Nanfang Hospital, Southern Medical University, 1838 Guangzhou North Avenue, Guangzhou, Guangdong 510515, P.R. China

E-mail: drjiang@163.com

E-mail: helenwxy@smu.edu.cn

*Contributed equally

Key words: colon capsule endoscopy, bowel preparation, polyethylene glycol, sodium phosphate incidence in many Asian countries in recent decades $(1,2)$. A recent study demonstrated that screening for CRC in average-risk adults was effective in reducing the mortality rate (3). A satisfactory screening method for CRC must be safe, non-invasive, cost-effective, easily acceptable and possess a high diagnostic accuracy (4).

The PillCam colon capsule endoscopy (CCE) may achieve direct visualization of the entire colon without sedation, radiation or air insufflation, which represents a non-invasive imaging system for exploring the colon (5). Although colonoscopy is currently considered to be the gold standard method for CRC screening and the diagnosis of most colonic diseases (4), the PillCam colon capsule endoscopy has been developed as the most promising approach to CRC screening in recent years, which is an alternative for patients with incomplete colonoscopy or who are reluctant to accept colonoscopy examination due to discomfort or embarrassment (6).

The effectiveness of CCE partly depends on the cleanliness of the colon. An optimal bowel preparation regimen for $\mathrm{CCE}$ is required for a clean intestine, capsule propulsion and visualization of the whole large intestine. Previous studies have demonstrated bowel preparation regimens for CCE that generally consist of a split-dosage of polyethylene glycol (PEG) with the volume of 41 , prokinetic agents and sodium phosphate (NaP) boosters (5,7-9). However, large volumes of laxatives may reduce patient compliance. Furthermore, NaP is associated with certain adverse events, such as electrolyte disturbance, acute nephropathy and kidney failure (10), and should be avoided in patients at increased risk of $\mathrm{NaP}$ toxicity. Therefore, there is a requirement for the current bowel preparation regimen to be improved.

The present study aimed to evaluate a novel low-volume and NaP-free bowel preparation regimen for CCE. In this regimen, the volume of PEG was reduced to 21 and NaP was substituted with PEG as the booster. Colon cleansing quality and completion rate were assessed.

\section{Patients and methods}

Patients. Between July 2013 and July 2014, a total of 31 patients were enrolled to the current prospective study at the Department of Gastroenterology, Nanfang Hospital, Southern Medical University (Guangzhou, China) in accordance with the 
Table I. Conventional (4) and novel bowel preparation regimens for colon capsule endoscopy.

Regimen

Time

Conventional

Novel

Day before examination

All day
$1 \mathrm{~h}$ before lunch
$1 \mathrm{~h}$ before supper
6:00-9:00 p.m.

Examination day

6:00 a.m.

6:00-7:00 a.m.

7:30 a.m.

7:45 a.m

8:00 a.m.

8:30 a.m.

9:30 a.m.

10:00 a.m.

12:30 p.m.

2:00 p.m.

3.00 p.m.

3:30 p.m.

4:30 p.m.
Clear liquids only

31 PEG

11 PEG

$20 \mathrm{mg}$ domperidone

Capsule ingestion

$45 \mathrm{ml} \mathrm{NaP}+11$ water

$30 \mathrm{ml} \mathrm{NaP}+11$ water

Snack (optional)

$10 \mathrm{mg}$ bisacodyl
Low-fiber diet

$5 \mathrm{mg}$ mosapride citrate

$5 \mathrm{mg}$ mosapride citrate

11 PEG

11 PEG

$5 \mathrm{mg}$ mosapride citrate

200 mg simethicone

Capsule ingestion

$0.51 \mathrm{PEG}$

0.51 PEG

Snack (optional)

PEG, polyethylene glycol; NaP, sodium phosphate.

following inclusion criteria: Aged 18-75 years, willing to accept CCE examination and providing signed informed consent. According to the criteria in a previously published study, exclusion criteria were as follows: Dysphagia or swallowing disorder, prior major abdominal surgery of the gastrointestinal tract, known or suspected bowel obstruction, presence of a cardiac pacemaker or other implanted electromedical devices and pregnancy (11). The methodology was approved by the Ethics Committee of Nanfang hospital.

PillCam colon capsule endoscopy. The PillCam CCE(PillCam COLON, Given Imaging; Medtronic, Dublin, Ireland) was $11 \times 31 \mathrm{~mm}$ in size and had two cameras, one at each end of the capsule, each capturing 2 images/sec. The angle of view for each imager was $156^{\circ}$. The capsule enters a $1 \mathrm{~h}$ delay mode following a 3-min initial function, then the system automatically restarts and functions for an additional $9 \mathrm{~h}$. Captured images were transmitted to the data recorder via eight sensors. A rapid real-time viewer allowed a real-time view during a PillCam procedure. Following the examination, the recorded data was downloaded into the Given Imaging RAPID 4 workstation (Medtronic). Captured videos were reviewed by two physicians who had prior experience with colonoscopy and small intestine capsule endoscopy. Colon cleansing levels were then assessed, as described below.

Bowel preparation regimens. As shown in Table I, the conventional regimen of bowel preparation for CCE includes 41 of an oral preparation of PEG (Colopeg; Roche Laboratories,
Gaillard, France), 1-2 oral boosters of $\mathrm{NaP}$ at a dosage of 30-45 ml (Fleet Phospho Soda ${ }^{\circledR}$; Wolf, Fleet, Lynchburg, VA, USA) and a suppository of bisacodyl as necessary (Dulcolax ${ }^{\circledR}$, Boehringer Ingelheim, Scherer, Aprilia, Italy) (4). In order to avoid adverse events associated with $\mathrm{NaP}$, improve patient compliance and maintain the quality of bowel cleansing, the current study evaluated a modified regimen. In the novel bowel preparation regimen, on one day prior to examination, a low-fiber diet was permitted, $5 \mathrm{mg}$ mosapride citrate (Gasmotin; Sumitomo Dainippon Pharma Co., Ltd., Osaka, Japan) was orally administered twice ( $1 \mathrm{~h}$ before lunch and $1 \mathrm{~h}$ before supper) and 11 of an oral preparation of PEG (Fortrans; Ipsen, Paris, France) at 6:00-9:00 p.m. On the day of the examination, an additional $11 \mathrm{PEG}, 5 \mathrm{mg}$ mosapride citrate and $200 \mathrm{mg}$ simethicone (Espumisan; Berlin-Chemie AG, Berlin, Germany) were orally administered at 2.5, 1 and $0.5 \mathrm{~h}$ prior capsule ingestion, respectively. PEG booster (0.5 1) was orally administered twice, 1 and $4 \mathrm{~h}$ following capsule ingestion. The detailed procedure of the novel regimen is presented in Table I. Patient education concerning the bowel preparation procedure for CCE was delivered by physicians prior to the examination, in order to improve the efficacy of the regimen. Any adverse effects were recorded, including nausea, vomiting, abdominal pain, dizziness, headache and allergy.

Performance evaluation. The four-point grading scale system reported by Leighton and Rex (12) was applied to evaluate colon cleansing levels. A 'poor' level of colon cleansing was 


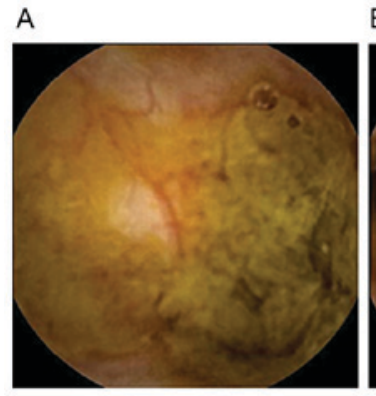

Poor
B

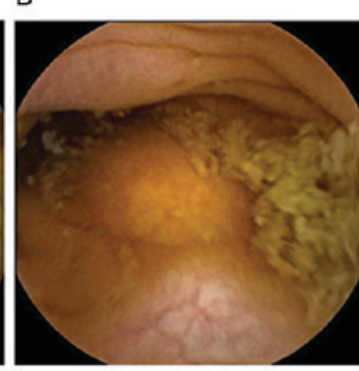

Fair
C

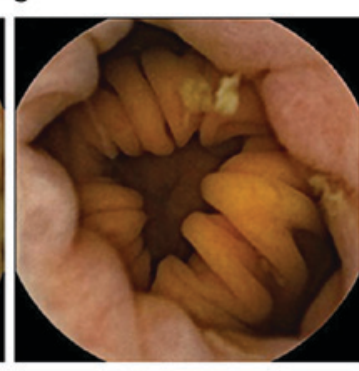

Good
D

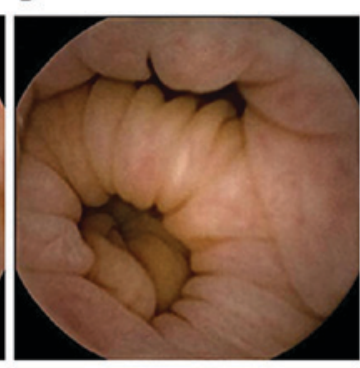

Excellent

Figure 1. Images of colon cleansing levels rated by a four-point scale grading system. Cleansing levels were rated as (A) poor, (B) fair, (C) good or (D) excellent.

Table II. Clinical indications of patients undergoing colon capsule endoscopy $(\mathrm{n}=33)^{\mathrm{a}}$.

\begin{tabular}{lcc}
\hline Clinical indication & $\mathrm{n}$ & Percentage \\
\hline Physical examination & 8 & 24.2 \\
Constipation & 6 & 18.2 \\
Abdominal pain & 5 & 15.2 \\
Diarrhea & 4 & 12.1 \\
Abdominal distension & 3 & 9.1 \\
Hematochezia & 2 & 6.1 \\
Acid reflux and heartburn & 2 & 6.1 \\
Recent change of bowel habits & 1 & 3.0 \\
Prior ulcer in terminal ileum & 1 & 3.0 \\
Crohn's disease & 1 & 3.0 \\
\hline
\end{tabular}

${ }^{\mathrm{a}} \mathrm{A}$ total of 29 patients were in the final analyses and as 4 of these patients suffered from two clinical indications, the sum of indications was 33 .

defined as a large amount of fecal residue. 'Fair' was defined as a sufficient amount of feces or turbid fluid to prevent reliable examination. 'Good' was defined as a small amount of feces or turbid fluid not interfering with examination. 'Excellent' was defined only small pieces of adherent feces (Fig. 1). For subsequent analysis, the grades of excellent and good were defined as 'adequate preparation', whereas fair and poor were defined as 'inadequate preparation'. The video footage was divided into five segments: Cecum, ascending colon, transverse colon, descending colon and rectosigmoid colon. Subsequently, overall cleansing levels and cleansing levels of each segment were assessed. Ileocecal valve transit time was defined as the time from capsule ingestion to the first image of the cecum. The examination was considered to be completed when the haemorrhoidal plexus was visualized.

\section{Results}

Study participants. A total of 29 patients were included in the final analysis (male/female, 13/16; mean age, 35 years; age range, $24-61$ years). Two patients (6\%) were excluded as data was not acquired due to technical failure. Major clinical indications included physical examination, constipation, abdominal pain and diarrhea (Table II). All enrolled patients

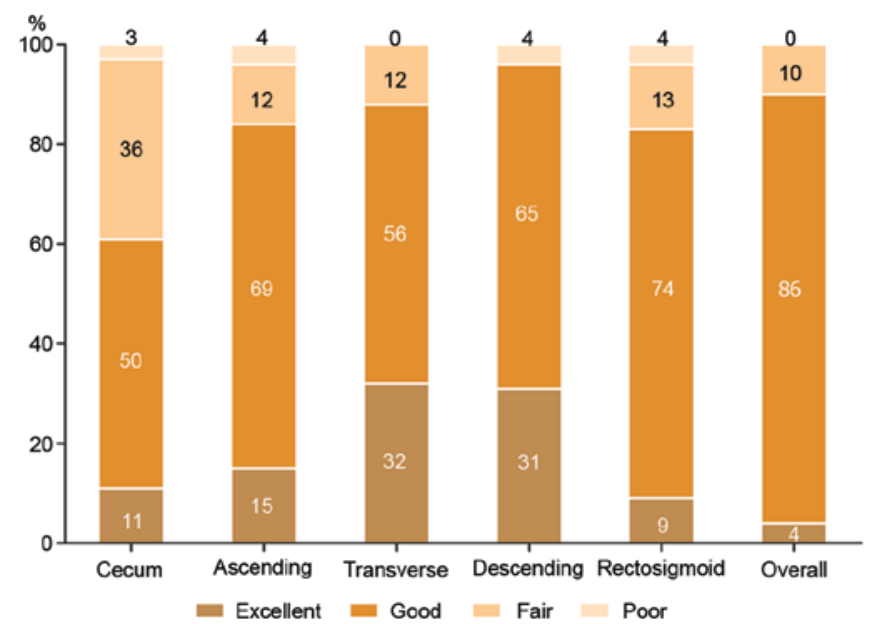

Figure 2. Colon cleansing levels in different colon segments and overall colon. Adequate levels (rated as good or excellent on a four-point scale grading system) were as follows: cecum, $61 \%$; ascending colon, $84 \%$; transverse colon, $88 \%$; descending colon, $96 \%$; rectosigmoid colon, $83 \%$; and overall colon, $90 \%$.

complied with the novel bowel preparation. No adverse effects associated with CCE occurred.

Colon cleansing levels. According to the four-point scale grading system, the results indicated that overall colon cleansing levels were rated as excellent in 1 patient (4\%), good in 25 patients $(86 \%)$ and fair in 3 patients $(10 \%)$. No patients were rated as poor (Fig. 2). In total, $90 \%$ of the patients exhibited adequate colon preparation (ratings of excellent or good). In the individual colon segments, the descending colonic mucosa exhibited the highest quality of cleansing (adequate cleansing level in $96 \%$ of patients), whereas the cecum exhibited the poorest quality (adequate cleansing level in 61\%). As for bubbles in the large intestine, overall the images were not evidently affected by bubbles and only a small number were observed (Fig. 2).

Colon capsule transmit time and completion rate. Ileocecal valve transit time (time from capsule ingestion to the first image of cecum) was $2.35 \pm 0.82 \mathrm{~h}$. Furthermore, 93.1\% (27/29) of capsules were located in the small intestine when they restarted following the $1 \mathrm{~h}$ delay mode. Two capsules were still in the stomach at this point and successfully travelled into the small intestine after the patients drank some water. Examination completion occurred in $79.3 \%$ (23/29) of patients. 
Table III. Location of colon capsule endoscopy at different time points $(n=29)$.

\begin{tabular}{llrr}
\hline Time & \multicolumn{1}{c}{ Location } & Patients (n) & Percentage \\
\hline Restart after 1 h delay & Stomach & 2 & 6.9 \\
& Small intestine & 27 & 93.1 \\
End of examination & Haemorrhoidal plexus (visible) & 23 & 79.3 \\
& Descending colon & 1 & 3.9 \\
& Transverse colon & 2 & 6.9 \\
& Ascending colon & 1 & 3.4 \\
\hline
\end{tabular}

At the end of CCE examination in 2 cases, the capsule was in the descending colon, in 1 case it was in the transverse colon, in 2 cases it was in the ascending colon and in 1 case it was in the cecum (Table III).

\section{Discussion}

At present, a standardized bowel preparation regimen is still not available. In previous studies, large volumes of PEG combined with a $\mathrm{NaP}$ booster regimen was most commonly applied (4,5,7-9). However, this remains controversial due to low patient compliance and safety concerns surrounding $\mathrm{NaP}(13,14)$. In the present study, a novel low-volume and NaP-free bowel preparation regimen was provided for CCE, which was demonstrated to be feasible.

Different bowel preparation regimens for CCE in previous studies have reported variable results; adequate colon cleansing ranged from 35 to $84 \%$ and completion rates ranged from 64 to $100 \%(4,7-9,14-24)$. More recently, modified regimens with low dosage PEG have been researched. Hartmann et al (14) reported that a 21 PEG plus ascorbic acid regimen yielded adequate cleansing levels in $82 \%$ of patients and Kakugawa et al (23) reported that a 2.3-2.6 1 polyethylene glycol electrolyte lavage solution (PEG-ELS) regimen yielded adequate cleansing levels in $94 \%$ of patients. However, Usui et al (24) evaluated a lower volume regimen with 0.71 PEG in second-generation colon capsule endoscopy, which yielded lower adequate cleansing levels of $60 \%$. According to the four-point grading scale system, $90 \%$ of patients achieved adequate colon cleansing level in the present study with a 21 PEG regimen. Consistent with the results reported by Spada et al (19), the current data revealed that the cecum was the segment with the lowest quality of cleansing (adequate level in 61\%) and the descending colon had the highest quality of cleansing (adequate level in 94\%). The current study demonstrated that a low-volume regimen could also achieve a high quality of colon cleansing.

A booster is necessary for cleaning the colonic mucosa and improving capsule excretion. The efficacy of $\mathrm{NaP}$ as booster has been reported in previous studies $(5,19,22)$, however it has associated adverse effects, including electrolyte disturbance, acute nephropathy and kidney failure. Guidelines established in Europe (UK National Patient Safety Agency and European Society of Gastrointestinal Endoscopy) advised against the routine use of $\mathrm{NaP}$ for bowel preparation due to safety concerns $(13,25)$, and an equivalent recommendation is suggested by the Guidelines published by Chinese society of digestive endoscopy in China (26). Therefore, there is an urgent requirement for a safe substitute for NaP. Without side effects such as electrolyte disturbance and renal damage, PEG has been demonstrated to be a safe booster. Spada et al (21) and Hartmann et al (14) reported PEG booster regimens obtained completion rate in 75 and $76 \%$ of cases, respectively. The regimen in the current study achieved a completion rate in $79.3 \%$ of cases. Compared with NaP-based regimens, the completion rate is lower, yet the application of PEG as booster is still promising for its safety and further studies should be performed to evaluate how completion rate may be increased. Previously, magnesium citrate was applied as an emerging booster with completion rates of $55-85 \%(23,24)$. These fluctuating results emphasize the need for further research on magnesium citrate as a booster.

There are other strategies that are favorable for improving the quality of colon cleansing and increasing completion rate. Several types of prokinetic agent have been used, such as tegaserod (8), domperidone $(4,6,15,18)$, metoclopramide (22) and mosapride $(23,27)$. Mosapride is an emerging prokinetic agent, working as a 5-hydroxytryptamine receptor 4 agonist and accelerating both gastric emptying and peristalsis of the small intestine (28). Wei et al (29) and Ida et al (30) recommended that patients received $10 \mathrm{mg}$ mosapride citrate 30-60 min prior to capsule ingestion. However, the dosage and administration time of mosapride remain to be evaluated. In the present study, to assist bowel cleansing and reduce the dosage of PEG, $5 \mathrm{mg}$ mosapride citrate was administered $1 \mathrm{~h}$ prior to lunch and supper on the day prior to examination and $1 \mathrm{~h}$ prior to capsule ingestion. The present results revealed that in 93.1\% (27/29) of patients, capsules were located in the small intestine when the capsules activated following the $1 \mathrm{~h}$ delay and the average ileocecal valve transit time was $2.35 \mathrm{~h}$. Although evidence from a controlled study is not available, this time was notably shorter than the 5-8 h exhibited in small intestine capsule endoscopy (31). In addition, simethicone is also considered to have an auxiliary effect for bowel preparation. In the studies of Kakugawa et al (23) and Usui et al (24), simethicone was applied, but its necessity was not discussed. In the present study, to decrease the number of bubbles in the large intestine, $200 \mathrm{mg}$ simethicone was ingested $30 \mathrm{~min}$ prior to capsule endoscopy. Ultimately, bisacodyl suppository was optional in previous studies $(4,14)$. In order to reduce the embarrassment 
of patients and maintain patient compliance, bisacodyl was not administered.

The present study demonstrated that a low-volume and NaP-free bowel preparation regimen was effective for colon capsule endoscopy. However, there were some limitations to the study, including the limited sample size and the absence of a controlled comparison with conventional colonoscopy. Furthermore, the study would be optimized if consecutive patients were included.

In conclusion, a novel low-volume and NaP-free bowel preparation regimen for $\mathrm{CCE}$ has been demonstrated to be feasible, with adequate colon cleansing levels and completion rate, and could therefore be used as an alternative regimen. Further studies should be performed in order to evaluate whether the completion rate can be increased, and the accuracy of CCE with the novel regimen should be compared with traditional colonoscopy in a randomized, controlled trial.

\section{References}

1. Ferlay J, Autier P, Boniol M, Heanue M, Colombet M and Boyle P: Estimates of the cancer incidence and mortality in Europe in 2006. Ann Oncol 18: 581-592, 2007.

2. Yeoh KG, Ho KY, Chiu HM, Zhu F, Ching JY, Wu DC, Matsuda T, Byeon JS, Lee SK, Goh KL, et al: The Asia-Pacific Colorectal Screening score: A validated tool that stratifies risk for colorectal advanced neoplasia in asymptomatic Asian subjects. Gut 60 1236-1241, 2011

3. Qaseem A, Denberg TD, Hopkins RH Jr, Humphrey LL, Levine J, Sweet DE and Shekelle P; Clinical Guidelines Committee of the American College of Physicians: Screening for colorectal cancer: A guidance statement from the American College of Physicians. Ann Intern Med 156: 378-386, 2012.

4. Schoofs N, Devière J and Van Gossum A: PillCam colon capsule endoscopy compared with colonoscopy for colorectal tumor diagnosis: A prospective pilot study. Endoscopy 38: 971-977, 2006.

5. Spada C, Hassan C, Galmiche JP, Neuhaus H, Dumonceau JM, Adler S, Epstein O, Gay G, Pennazio M, Rex DK, et al: Colon capsule endoscopy: European society of gastrointestinal endoscopy (ESGE) Guideline. Endoscopy 44: 527-536, 2012.

6. Triantafyllou K, Viazis N, Tsibouris P,Zacharakis G, Kalantzis C, Karamanolis DG and Ladas SD: Colon capsule endoscopy is feasible to perform after incomplete colonoscopy and guides further workup in clinical practice. Gastrointest Endosc 79: 307-316, 2014

7. Van Gossum A, Munoz-Navas M, Fernandez-Urien I, Carretero C, Gay G, Delvaux M, Lapalus MG, Ponchon T, Neuhaus H, Philipper M, et al: Capsule endoscopy versus colonoscopy for the detection of polyps and cancer. N Engl J Med 361: 264-270, 2009

8. Eliakim R, Fireman Z, Gralnek IM, Yassin K, Waterman M, Kopelman Y, Lachter J, Koslowsky B and Adler SN: Evaluation of the PillCam Colon capsule in the detection of colonic pathology: Results of the first multicenter, prospective, comparative study. Endoscopy 38: 963-970, 2006.

9. Eliakim R, Yassin K, Niv Y, Metzger Y, Lachter J, Gal E, Sapoznikov B, Konikoff F, Leichtmann G, Fireman Z, et al: Prospective multicenter performance evaluation of the second-generation colon capsule compared with colonoscopy. Endoscopy 41: 1026-1031, 2009.

10. Wexner SD, Beck DE, Baron TH, Fanelli RD, Hyman N, Shen B and Wasco KE; American Society of Colon and Rectal Surgeons; American Society for Gastrointestinal Endoscopy; Society of American Gastrointestinal and Endoscopic Surgeons: A consensus document on bowel preparation before colonoscopy: Prepared by a task force from the American society of colon and rectal surgeons (ASCRS), the American society for gastrointestinal endoscopy (ASGE), and the Society of American gastrointestinal and endoscopic surgeons (SAGES). Gastrointest Endosc 63: 894-909, 2006.

11. Ladas SD, Triantafyllou K, Spada C, Riccioni ME, Rey JF, Niv Y, Delvaux M, de Franchis R and Costamagna G; ESGE Clinical Guidelines Committee: European society of gastrointestinal endoscopy (ESGE): Recommendations (2009) on clinical use of video capsule endoscopy to investigate small-bowel, esophageal and colonic diseases. Endoscopy 42: 220-227, 2010.
12. Leighton JA and Rex DK: A grading scale to evaluate colon cleansing for the PillCam COLON capsule: A reliability study. Endoscopy 43: 123-127, 2011.

13. Hassan C, Bretthauer M, Kaminski MF, Polkowski M, Rembacken B, Saunders B, Benamouzig R, Holme O, Green S, Kuiper T, et al: Bowel preparation for colonoscopy: European Society of Gastrointestinal Endoscopy (ESGE) Guideline. Endoscopy 45: 142-150, 2013.

14. Hartmann D, Keuchel M, Philipper M, Gralnek IM, Jakobs R, Hagenmüller F, Neuhaus H and Riemann JF: A pilot study evaluating a new low-volume colon cleansing procedure for capsule colonoscopy. Endoscopy 44: 482-486, 2012.

15. Gay G, Delvaux M, Frederic M and Fassler I: Could the colonic capsule PillCam Colon be clinically useful for selecting patients who deserve a complete colonoscopy?: Results of clinical comparison with colonoscopy in the perspective of colorectal cancer screening. Am J Gastroenterol 105: 1076-1086, 2010.

16. Pilz JB, Portmann S, Peter S, Beglinger C and Degen L: Colon capsule endoscopy compared to conventional colonoscopy under routine screening conditions. BMC Gastroenterol 10: 66, 2010.

17. Sacher-Huvelin S, Coron E, Gaudric M,Planche L, Benamouzig R, Maunoury V, Filoche B, Frédéric M, Saurin JC, Subtil C, et al: Colon capsule endoscopy vs. colonoscopy in patients at average or increased risk of colorectal cancer. Aliment Pharmacol Ther 32: 1145-1153, 2010.

18. Sieg A, Friedrich K and Sieg U: Is PillCam COLON capsule endoscopy ready for colorectal cancer screening? A prospective feasibility study in a community gastroenterology practice. Am J Gastroenterol 104: 848-854, 2009.

19. Spada C, Hassan C, Ingrosso M, Repici A, Riccioni ME Pennazio M, Pirozzi GA, Pagano N, Cesaro P, Spera G, et al: A new regimen of bowel preparation for PillCam colon capsule endoscopy: A pilot study. Dig Liver Dis 43: 300-304, 2011.

20. Spada C, Hassan C, Munoz-Navas M, Neuhaus H, Deviere J, Fockens P, Coron E, Gay G, Toth E, Riccioni ME, et al: Secondgeneration colon capsule endoscopy compared with colonoscopy. Gastrointest Endosc 74: 581-589.e1, 2011.

21. Spada C, Riccioni ME, Hassan C, Petruzziello L, Cesaro P and Costamagna G: PillCam colon capsule endoscopy: A prospective, randomized trial comparing two regimens of preparation. J Clin Gastroenterol 45: 119-124, 2011.

22. Sung J, Ho KY, Chiu HM, Ching J, Travis S and Peled R: The use of Pillcam Colon in assessing mucosal inflammation in ulcerative colitis: A multicenter study. Endoscopy 44: 754-758, 2012.

23. Kakugawa Y, Saito Y, Saito S, Watanabe K, Ohmiya N, Murano M, Oka S, Arakawa T, Goto H, Higuchi K, et al: New reduced volume preparation regimen in colon capsule endoscopy. World J Gastroenterol 18: 2092-2098, 2012

24. Usui S, Hosoe N, Matsuoka K, Kobayashi T, Nakano M, Naganuma M, Ishibashi Y, Kimura K, Yoneno K, Kashiwagi K, et al: Modified bowel preparation regimen for use in secondgeneration colon capsule endoscopy in patients with ulcerative colitis. Dig Endosc 26: 665-672, 2014.

25. Connor A, Tolan D, Hughes S, Carr N and Tomson C: Consensus guidelines for the safe prescription and administration of oral bowel-cleansing agents. Gut 61: 1525-1532, 2012.

26. Chinese Society of Digestive Endoscopy: Chinese guidelines for bowel preparation of digestive endoscopy (Draft). Chin J Dig Endosc 30: 481-483, 2013.

27. Hosoe N, Matsuoka K, Naganuma M, Ida Y, Ishibashi Y, Kimura K, Yoneno K, Usui S, Kashiwagi K, Hisamatsu T, et al: Applicability of second-generation colon capsule endoscope to ulcerative colitis: A clinical feasibility study. J Gastroenterol Hepatol 28: 1174-1179, 2013.

28. Okamura K, Sasaki N, Yamada M, Yamada H and Inokuma H: Effects of mosapride citrate, metoclopramide hydrochloride, lidocaine hydrochloride, and cisapride citrate on equine gastric emptying, small intestinal and caecal motility. Res Vet Sci 86: 302-308, 2009

29. Wei W, Ge ZZ, Lu H, Gao YJ, Hu YB and Xiao SD: Effect of mosapride on gastrointestinal transit time and diagnostic yield of capsule endoscopy. J Gastroenterol Hepatol 22: 1605-1608, 2007.

30. Ida $\mathrm{Y}$, Hosoe $\mathrm{N}$, Imaeda $\mathrm{H}$, Bessho R, Ichikawa R, Naganuma $\mathrm{M}$, Kanai T, Hibi T and Ogata H: Effects of the oral administration of mosapride citrate on capsule endoscopy completion rate. Gut Liver 6: 339-343, 2012.

31. Selby W: Complete small-bowel transit in patients undergoing capsule endoscopy: Determining factors and improvement with metoclopramide. Gastrointest Endosc 61: 80-85, 2005. 